\title{
Archéométrie et histoire des techniques : les procédés direct et indirect en sidérurgie ( $\mathrm{XIV}^{\mathrm{e}}$ $\mathrm{XVII}{ }^{\mathrm{e}}$ siècle)
}

Archaeometry and History of Technonogy: Direct and Indirect Processes in Iron Metallurgy (14th-17th centuries)

\section{Philippe Dillmann et Maxime L'Héritier}

\section{(2) OpenEdition}

\section{Journals}

Édition électronique

URL : http://journals.openedition.org/artefact/331

DOI : 10.4000/artefact.331

ISSN : 2606-9245

Éditeur :

Association Artefact. Techniques histoire et sciences humaines, Presses universitaires du Midi

Édition imprimée

Date de publication : 1 octobre 2016

Pagination : 63-81

ISBN : 978-2-7535-5174-9

ISSN : 2273-0753

Référence électronique

Philippe Dillmann et Maxime L'Héritier, « Archéométrie et histoire des techniques : les procédés direct et indirect en sidérurgie (XIVe-XVIIe siècle) », Artefact [En ligne], 4 | 2016, mis en ligne le 07 juillet 2017, consulté le 23 avril 2019. URL : http://journals.openedition.org/artefact/331 ; DOI : 10.4000/ artefact.331 


\section{Archéométrie et histoire des techniques: les procédés direct et indirect en sidérurgie ( $\mathrm{XIV}^{\mathrm{e}}-\mathrm{xVII}{ }^{\mathrm{e}}$ siècle)}

Philippe DILLMANN* et Maxime L'HérITIER **

\section{Résumé}

Cet article présente l'apport des méthodes archéométriques à la réflexion en histoire des techniques. Il prend pour sujet la métallurgie des métaux ferreux pour laquelle une mutation importante des filières techniques apparaît et se répand dans le nord de l'Europe entre la fin du Moyen Âge et le début de l'époque moderne. L'analyse des fers de renfort employés dans les grands monuments religieux et civils construits à cette période a permis de pister l'apparition et la diffusion du procédé indirect pour la fabrication de ces produits métalliques. Elle montre pour ce marché un rythme de diffusion du nouveau procédé différent de celui que révèlent les sources écrites pour la production du métal. Ces résultats interrogent la nature spécifique de ce marché mais également celle de la diffusion de l'innovation liée à l'apparition du nouveau procédé.

Mots-clefs : archéométrie, innovation, procédé indirect, sidérurgie.

\section{Abstract. Archaeometry and history of technonogy : direct and indirect processes in iron metallurgy (14th-17th centuries)}

This paper aims to present the contribution of archaeometric approaches to the reflexion in history of technology. This will be illustrated by the case of ferrous metals. Between the end of the Middle Ages and the beginning of the modern period, a new ironmaking process appeared and spread in the north of Europe. The analysis of reinforcing ferrous elements employed for the building of religious and civil monuments at this period allowed us to follow the diffusion of

* LAPA-IRAMAT, NIMBE,CEA, CNRS, université Paris-Saclay, CEA Saclay 91191 Gif-sur-Yvette France. Contact [philippe.dillmann@cea.fr/].

** Université Paris 8, département d'histoire, EA 1571 HPSS. Contact [maxime.1_heritier@univ-paris8. $\mathrm{fr}]$. 
the indirect process for the making of these metallic products. It shows for this market, a pace of diffusion, different from the one suggested by written sources for the production process. This discrepancy is discussed considering the specificity of this market as well as the diffusion of the innovation linked to the appearance of the indirect process.

\section{Keywords : archaeometry, indirect process, innovation, iron and steel making.}

L'apparition et la diffusion d'un nouveau procédé de production des métaux ferreux (le procédé dit « indirect ») à la fin du Moyen Âge et au début de l'époque moderne a été, et reste aujourd'hui, un sujet de premier plan pour l'histoire des techniques. En effet, cette mutation, utilisant la force hydraulique qui se répand au Moyen Âge, permet une production de grande ampleur des métaux ferreux à l'époque moderne. Au xviII siècle, ses formes "stabilisées", procédés wallon et comtois, sont installées dans tout le nord de l'Europe, mais restent peu répandues dans certaines régions méridionales. L'étude de la diffusion de cette innovation et de ses modes de substitution ou de superposition aux procédés déjà en place revêt un intérêt particulier en histoire des techniques car il touche à de nombreuses notions clés : transmission des savoirs et des savoir-faire, définition de l'innovation, évolutions et adaptations des systèmes techniques. Les sources écrites exploitées depuis plusieurs dizaines d'années par les chercheurs offrent un certain nombre d'indices pour traiter de ces aspects. Elles ne sont cependant pas sans ambiguïté quant à la définition exacte et précise des techniques et des procédés mentionnés. Par ailleurs, ne révélant bien souvent que la première mention des procédés, le temps de l'irruption de l'innovation ${ }^{1}$, elles ne permettent de donner qu'une vision parcellaire de la réalité technique et en particulier de celle liée à la matérialité. Les fouilles archéologiques de certains ateliers de production du fer permettent quant à elles de caractériser en partie ces aspects techniques, mais sont encore trop rares et circonscrites pour avoir une perception fine de la diffusion de cette innovation sur un territoire.

L'archéométrie, qui fait partie intégrante des sciences archéologiques, est un domaine de recherche fortement interdisciplinaire qui s'intéresse aux informations enregistrées par les objets anciens, artefacts ou archives environnementales, à différentes échelles, le plus souvent observables à travers la mesure instrumentée de paramètres inaccessibles à l'observation visuelle. Ses méthodes relèvent de disciplines des sciences chimiques et physiques, sciences de la Terre et de la Vie et des sciences environnementales. Ses objectifs sont de fournir un nouveau type de source dans le cadre des problématiques historiques. Ces dernières années, dans le domaine de l'étude de la métallurgie du fer, la discipline a vu des avancées méthodologiques significatives, en particulier pour la détermination des procédés de fabrication et des filières techniques, mais également pour l'étude de la circulation des matériaux et des objets ou leur datation ${ }^{2}$. Ces avancées permettent aujourd'hui de compléter les sources archéologiques 
et historiques par l'étude des objets et de leurs matières constitutives. Les méthodes de l'archéométrie des objets ferreux sont donc aujourd'hui " naturellement » intégrées aux démarches interdisciplinaires des travaux en histoire des techniques sidérurgiques pratiquées par différentes équipes.

Nous allons présenter dans cet article comment les approches archéométriques développées dans le cadre de l'étude de la transition entre les filières de production en sidérurgie à la fin du Moyen Âge et à l'époque moderne, apportent un éclairage nouveau et complémentaire aux données archéologiques et aux sources écrites, qui permet de nuancer la perception que l'on avait jusqu'alors de l'installation du nouveau procédé dans un territoire technique correspondant au nord de la France et à la Belgique actuelle.

\section{Définitions technique et métallurgique des filières sidérurgiques}

Le fer n'existe pas à l'état natif sur terre. Il est associé à des oxydes, des sulfures ou des carbonates, formes thermodynamiquement plus stables qui, combinées à une gangue de composés ne contenant pas de fer, constituent le minerai. La transformation du minerai en métal se fait grâce à une opération technique, la réduction ${ }^{3}$ qui a lieu dans un fourneau qui a changé de dimensions et de forme au cours de l'histoire de la sidérurgie ${ }^{4}$. L'opération utilise la combustion du charbon de bois qui s'associe à l'oxygène de l'air introduit dans le fourneau par une ventilation pour former du monoxyde puis du dioxyde de carbone et permet de " libérer " l'élément métallique.

La première filière à être apparue en sidérurgie est dite directe. Fondée sur l'utilisation de fourneaux de taille relativement modeste, les bas fourneaux, elle permet de transformer le minerai en métal en une opération unique. La ventilation est d'abord naturelle puis méca- nique, associée à la force manuelle, puis, dès la fin du XIII ${ }^{e}$ siècle, à l'utilisation de la force hydraulique pour actionner des soufflets. Nous reviendrons plus en détail sur l'historique de l'apparition de cette force hydraulique pour la ventilation du fourneau. D'un point de vue purement métallurgique, cette ventilation, avant sa mécanisation hydraulique, permet d'atteindre les conditions thermodynamiques $^{5}$ de transformation du minerai en métal mais pas celle de sa fusion ${ }^{6}$. Il en résulte que le métal est obtenu sous la forme d'une masse hétérogène ${ }^{7}$, piégeant un certain nombre d'impuretés. Ces impuretés, appelées inclusions, sont en fait constituées de composés du minerai, initialement présents dans la gangue, qui ne sont pas transformés en métal et dont la plus grande partie est évacuée sous forme de scories pendant l'opération de réduction. L'hétérogénéité de la masse de métal obtenue avec le procédé direct réside également dans le fait que la teneur en carbone, et donc la 
quantité $\mathrm{d}^{\prime}$ acier ${ }^{8}$, est répartie de manière variable au sein du métal. Cette masse de métal est sortie du bas fourneau puis martelée, en suivant une chaîne opératoire qui peut être divisée spatialement, afin d'obtenir des demi-produits puis des produits finis.

La généralisation de l'usage de l'énergie hydraulique au Moyen Âge', conduit à son utilisation pour la ventilation des fourneaux. Ceci est à l'origine d'une montée en température qui, associée à une plus grande diffusion du carbone provenant du charbon de bois au sein du métal, permet d'atteindre sa température de fusion et d'obtenir un nouveau matériau : la fonte, alliage ferreux contenant plus de $2 \%$ de carbone ${ }^{10}$. Cet alliage dispose d'un point de fusion plus bas que le fer et les aciers et peut ainsi être moulén ${ }^{11}$. La fonte est cependant plus fragile (cassante) que ces derniers et ne peut donc remplir les mêmes usages. Il est alors nécessaire de le transformer au cours d'une seconde étape, l'affinage, destinée à retirer une partie du carbone pour obtenir, en fin d'opération, $\mathrm{du}$ fer ou de l'acier. C'est la raison pour laquelle cette nouvelle filière qui apparaît et se répand en Europe dans les derniers siècles du Moyen Âge et au début de l'époque moderne, est nommée " procédé indirect ». Il est à noter que l'affinage se déroule, jusque dans la seconde moitié du xIx ${ }^{\mathrm{e}}$ siècle, en dessous de la température de fusion des fers et des aciers. Il en résulte que, comme pour le procédé direct, c'est une masse de métal à l'état solide qui est produite à l'issue de l'opération d'affinage. Cette masse, ici encore, piège un certain nombre d'impuretés sous la forme d'inclusions, issues principalement de l'oxydation de certains éléments de la fonte. Ces inclusions ont donc une composition différente de celles produites lors du procédé direct et c'est leur analyse qui permet de distinguer les métaux issus des deux filières.

\section{Brève historiographie de l'apparition et de la diffusion de la filière indirecte}

En métallurgie du fer, l'usage de la force hydraulique pour actionner la soufflerie est postérieur à son application au martelage, dont les origines remontent au $\mathrm{XII}^{\mathrm{e}}$ siècle ${ }^{12}$. L'arc alpin est un des foyers de cette nouveauté. Les termes de furnus et de fusina appliqués dès le XIII ${ }^{\mathrm{e}}$ siècle à la métallurgie du fer désignent les deux composantes d'un atelier fonctionnant à l'énergie hydraulique ${ }^{13}$. Le plus ancien couple furnus / fusina recensé pour le travail du fer remonte en effet à la période
1269-1272 dans le territoire alpin de la Valteline $^{14}$. La terminologie associée aux produits de ces ateliers, ferrum crudum et ferrum coctum, a conduit certains chercheurs à formuler l'hypothèse que ce procédé produisant du fer en deux étapes était l'ancêtre du procédé indirect ${ }^{15}$. Rien n'indique toutefois que la production de fonte était habituelle dans ces établissements sidérurgiques ${ }^{16}$, même si le jeu délicat de la soufflerie hydraulique pouvait assurément amener à la production 
ponctuelle, contrôlée ou non, de petites quantités de fonte si le fourneau montait trop en température ${ }^{17}$. La complexité de ce dossier alpin ne peut être résumée $\mathrm{ici}^{18}$, mais le ferrum crudum et le ferrum coctum issus respectivement du furnus et de la fusina semblent bien faire référence à des produits spécifiques, voire nouveaux ${ }^{19}$. L'application de la force hydraulique au procédé direct pouvait en effet viser à obtenir une grosse masse de fer solide, afin de tirer parti de son hétérogénéité pour produire différentes qualités de fer et d'acier forgeables ${ }^{20}$. En l'état actuel de la recherche, les ateliers de Bornio et Semogo dans la Valteline restent toutefois les plus anciens exemples documentés d'utilisation de la soufflerie hydraulique en procédé direct, bien que, selon Catherine Verna, il faille davantage y voir la première mention de cette innovation plutôt que son "foyer initial $^{21} »$. Un tel usage de l'énergie hydraulique pour activer les soufflets de bas fourneaux se retrouve entre le début du $x_{I V}{ }^{e}$ et le début du Xve siècle dans plusieurs régions montagneuses à tradition métallurgique. C'est en particulier le cas de la mouline pyrénéenne ou languedocienne, mais également du martinetus dauphinois qui adoptent progressivement la soufflerie hydraulique en plus du gros marteau servant à battre le fer ${ }^{22}$. Cette nouvelle application de l'énergie hydraulique pose bien évidemment des questions techniques. Maintenir une température de fusion inférieure à celle $\mathrm{du}$ fer pour rester dans le domaine des aciers et éviter celui de la fonte liquide devait nécessiter une certaine maîtrise de la conduite du foyer et des adaptations de la forme du fourneau ${ }^{23}$.
À la même époque, le procédé indirect impliquant hauts fourneaux et affineries et faisant également appel à la soufflerie hydraulique, cette fois pour fondre le fer, est attesté dans plusieurs régions du nord de l'Europe. Les plus anciennes évidences de ce nouveau procédé sont ici archéologiques : en Suède dans le Bergsland et en Allemagne, dans le comté de la Marck. En Suède, dans le district de Norberg, Gert Magnusson a mis au jour un haut fourneau sur le site de Lapphyttan, associé à huit petits fourneaux manuels dédiés à l'affinage de la fonte ${ }^{24}$. La datation par thermoluminescence du fourneau a livré une date comprise entre 1279 et 1390 pour sa dernière période de chauffe ${ }^{25}$. D'autres sites sidérurgiques ont également été découverts (Vinarhyttan, Moshyttan) et, les datations précises de ces ensembles ayant longtemps été controversées, certains auteurs n'hésitent pas à prendre les bornes chronologiques les plus hautes pour évoquer une apparition de l'innovation dès les $\mathrm{XI}^{\mathrm{e}}$-XIII ${ }^{\mathrm{e}}$ siècle ${ }^{26}$ ! De récentes études sédimentologiques sur le haut fourneau de Moshyttan, un temps passé pour être le plus ancien et remonter au $\mathrm{XI}^{\mathrm{e}}$ siècle, placent désormais son installation dans la seconde moitié du XIII $^{\mathrm{e}}$ siècle $^{27}$. En Allemagne, plusieurs fouilles ont mis au jour les vestiges de hauts fourneaux (Flossöfen) datés des $\mathrm{XIII}^{\mathrm{e}}$-XIV ${ }^{\mathrm{e}}$ siècle et associés à la présence de laitiers, scories légères caractéristiques de la production de fonte ${ }^{28}$. Dans la vallée de la Volme, le site de Haus Rhade ayant fonctionné entre le $\mathrm{XIII}^{\mathrm{e}}$ et le début $d u \mathrm{xv}^{\mathrm{e}}$ siècle a également révélé la présence de scories lourdes identifiées comme des scories d'affinage de la fonte ${ }^{29}$. Le site de Jubachtal montre 
quant à lui un fourneau connecté à plusieurs bas foyers d'affinage à l'instar du site du Lappythan. Ces premiers hauts fourneaux semblent toutefois avoir pu produire alternativement des masses de fer forgeable ou de la fonte liquide. À Kerspetalsperre, où deux hauts fourneaux ont été datés par radiocarbone ${ }^{30}$, les archéologues proposent de restituer une transition technique entre un procédé direct plus ancien (Rennöfen, VIII $^{\mathrm{e}}$-XIII ${ }^{\mathrm{e}}$ siècle) et les hauts fourneaux

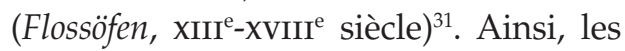
données archéologiques concordent pour placer le développement des premiers hauts fourneaux dans la seconde moitié du $\mathrm{XIII}^{\mathrm{e}}$ siècle de manière concomitante dans plusieurs régions d'Europe du Nord, au moment où l'adaptation de la soufflerie hydraulique se fait également pour le procédé direct.

Ces quelques exemples archéologiques, s'ils témoignent de l'implantation du nouveau procédé dans certaines régions, éclairent mal sa diffusion. Dans le royaume de France, le plus ancien fourneau fouillé est celui de Glinet dans le pays de Bray, daté du début de la période moderne (1480-1580) ${ }^{32}$. L'enquête dans les archives, bien plus vaste, vient en partie compléter cette chronologie, mais doit être entreprise avec prudence, tant les mots revêtent parfois des réalités différentes suivant les régions ${ }^{33}$. Ainsi, dans le comté de Namur, l'installation de sept forges à Marches-les-Dames à l'initiative du comte Guillaume et le développement d'établissements sidérurgiques dans la région laissent transparaître un nouveau vocabulaire. Il ne qualifie pas, dans un premier temps, les installations de forge elles-mêmes, mais les artisans de «fondeurs » ou " affi- neurs ", travaillant dans une des sept forges de Marches-les-Dames, mais aussi à Jausse (1356) et entre Sambre et Meuse, à Ermeton (1370) $)^{34}$. Plusieurs sont d'origine allemande. D'après Brian Awty, la date de l'introduction du procédé indirect dans la région n'est pas figée, mais tous les indices concordent pour affirmer que la production de fer et celle de plomb étaient liées dès les années 1340 dans les environs de Namur et que les structures métallurgiques nécessaires pour fondre le fer en fonte étaient alors certainement disponibles ${ }^{35}$. Il s'interroge notamment sur une mention de redevance à Marche, datée de 1345, en "fer de plokestoire", $q^{\prime}$ 'il propose de traduire par « fer fondu à la manière du plomb ${ }^{36} »$. En Lorraine, l'hypothèse d'une introduction précoce du nouveau procédé a été avancée par Alain Girardot sur la base de deux corpus de textes : un contrat de 1323 et des comptabilités de 1324-1327 relatives à la construction d'une "forge faisant feir par yawe » à Moyeuvre et le contrat d'affermage d'une forge hydraulique contenant deux roues à Champigneulles en $1391^{37}$. Le caractère hydraulique des installations ne fait aucun doute, mais une relecture plus fine des sources est plus prudente sur leur interprétation : l'application de l'hydraulique aux soufflets ne semble désormais plus nécessairement assurée et la terminologie associée au procédé indirect n'est pas complète et ne permet pas de conclure à l'installation d'un tel atelier à cette époque ${ }^{38}$. Les premières évidences incontestables de l'implantation du nouveau procédé dans le royaume de France remontent à la fin du xIV ${ }^{e}$ siècle dans le pays d'Othe, entre Troyes et Sens. Nicolas de Fontenay, officier du duc de Bourgogne et seigneur 
de Saint-Liébaut, y fait construire en 1377 une " forge à affiner fer » équipée d'une roue actionnant de "grans souflez $^{39}$ ", termes qui évoquent cette fois explicitement l'affinerie, seconde étape du procédé indirect. Dans le comté de Nevers, récente acquisition du duc de Bourgogne, on trouve vingt ans plus tard des mentions de "fondoère » qualifiant expressément l'installation sidérurgique à Jouet-sur-l'Aubois (1401) et Précy (1402). Comme en pays namurois, la présence de maîtres de forge allemands - mais aussi wallons - ayant contribué à diffuser l'innovation est manifeste, en particulier Coleçon le Liégeois à SaintLiébaut ou encore Haynemant et Henry Le Alemand respectivement affineur et fondeur à la forge de Jouet-sur-l'Aubois en $1402^{40}$. B. Awty souligne l'importance de ces flux de populations dans l'introduction du nouveau procédé ${ }^{41}$. Les évidences $\mathrm{du} \mathrm{Xv}^{\mathrm{e}}$ siècle traduisent encore davantage la réalité technique des ateliers de production. En Haute-Marne, une forge permettant de "fondre, affiner et forgier ${ }^{42}$ » est construite dans l'abbaye bénédictine de Bèze en 1427. Dans le Jura voisin, les premières occurrences de « haut fornel » ou de "ferrière ", désignant l'ensemble de l'exploitation, remontent au milieu du $\mathrm{Xv}^{\mathrm{e}}$ siècle (1448 à $\left.J_{o u x}{ }^{43}\right)$. Enfin, la première mention indiscutable d'une affinerie en Lorraine date de 1445-1446 à la forge de Vaux dans le Pays Haut et celle d'un " hault fournelz » de 1495 à la forge de Sexey ${ }^{44}$. En Champagne, à partir du milieu du $x^{\text {e }}$ siècle, l'abbaye de Clairvaux participe du développement du nouveau procédé en parallèle des investisseurs laïcs, avec les forges de Montheries, puis de Champigny et de Bailly-aux-Forges : les baux y donnent une description précise des installations ${ }^{45}$. La chronologie normande est similaire, avec la forge du Becquet prise à cens en 1451 par Henri le Marteleur, Pierre le Fondeur, du pays de Liège, et Henry le Féron, du comté de Namur, pour y construire un "moulin à fer », suivi de l'aménagement en amont d'un «sault d'eaue a edifier une fonderie ou ferronerie » en $1454^{46}$. Les occurrences se multiplient dans l'ensemble de ces régions à partir de la seconde moitié $\mathrm{du}$ $\mathrm{XV}^{\mathrm{e}}$ siècle et les premiers hauts fourneaux sont attestés en Angleterre au tournant des $\mathrm{XV}^{\mathrm{e}}$ et $\mathrm{XVI}^{\mathrm{e}}$ siècles ${ }^{47}$. Plus au sud, dans les Alpes italiennes, les premières certitudes remontent à la fin $\mathrm{du} \mathrm{XV}^{\mathrm{e}}$ ou au $\mathrm{XVI}^{\mathrm{e}}$ siècle, où plusieurs vallées alpines se spécialisent dans la production de fonte et de boulets ${ }^{48}$. De l'autre côté des Alpes, le nouveau procédé ne s'implante véritablement en Dauphiné qu'au début du $\mathrm{XVI}^{\mathrm{e}}$ siècle $^{49}$. Des spécialistes bergamasques contribuent également au développement de cette nouvelle sidérurgie dans les Apennins vers 1540.

Le propre des hauts fourneaux est de fonctionner en continu et de permettre la coulée de plusieurs gueuses de fonte par jour. Mais augmentent-ils considérablement les quantités de métal produites? Si on ignore les capacités de production de ces premiers hauts fourneaux, au tournant des $\mathrm{XV}^{\mathrm{e}}$ et $\mathrm{XVI}^{\mathrm{e}}$ siècles, la production d'une tonne de fonte par jour semble habituelle sous forme de plusieurs gueuses de fonte de 150 à $500 \mathrm{~kg}$. Par ailleurs, il ne faut pas oublier que, pour être transformée en fer ou en acier, cette fonte doit être affinée dans une opération qui pourrait potentiellement limiter la productivité, au moins aux origines du procédé. Ces quantités 
semblent supérieures à ce que l'on peut reconstituer des forges hydrauliques du XIV $^{e}$ siècle où la production hebdomadaire de fer semble comprise entre $500 \mathrm{~kg}$ et une tonne, tant à Albiès dans les Pyrénées qu'à Byrkenott en Angleterre ${ }^{50}$. En revanche, des exemples plus tardifs, comme les Radwerk de Styrie ou les ferrières de Plaisance au $\mathrm{xv}^{\mathrm{e}}$ siècle, semblent capables de fournir 600 à $700 \mathrm{~kg}$ de masses de fer ou d'acier par jour, qu'il convient de trier et d'épurer.

La conjonction de l'archéologie et de l'enquête dans les archives permet de cerner les débuts de l'innovation à l'échelle de plusieurs régions d'Europe occidentale. Elle reste en revanche plus évasive sur son expansion et tend presque à donner l'impression d'une installation rapide, hégémonique et durable du nouveau procédé suite à ses premières occurrences dans un territoire : en Allemagne, dès la fin du XIII ${ }^{\mathrm{e}}$ siècle, en Wallonie dès le milieu du xIv ${ }^{e}$ siècle, et dans certaines régions du royaume de France à partir de la fin du xIv ${ }^{\mathrm{e}}$ siècle. Pourtant, ce n'est pas tant l'irruption de l'innovation qui importe, mais bien sa diffusion, le moment où ses produits, par l'ampleur qu'ils prennent sur les marchés à l'échelle d'un territoire donné, lui donnent une influence majeure sur la société51. L'étude archéométrique des matériaux eux-mêmes, produits par ces différentes filières techniques permet alors d'apporter une autre vision diachronique de la diffusion de l'innovation à la fin du Moyen Âge et au début de la période moderne.

\section{Apport méthodologique de l'archéométrie : lire les procédés dans la matière}

Comme cela a été expliqué, les fers et les aciers obtenus par les deux filières piègent en leur sein une quantité importante d'inclusions non métalliques issues de l'opération de réduction. Ces inclusions sont de très petite taille (de l'ordre de quelques dizaines de micromètres). Des travaux relativement récents ${ }^{52}$ ont permis de montrer qu'il était possible de différencier de manière statistique la composition des inclusions des deux filières. Un corpus de référence constitué d'objets archéologiques issus de manière certaine de la filière directe ou de la filière indirecte a été collecté. La composition des inclusions de ces objets a pu être ana- lysé $e^{53}$. Par une approche statistique sur les résultats de ces analyses, les objets de la filière directe et ceux de la filière indirecte ont pu être nettement séparés ${ }^{54}$. Cela est dû notamment au fait que certains éléments sont éliminés de la chaîne opératoire en passant dans le laitier ${ }^{55}$ et se retrouvent en quantité moindre dans les inclusions produites par le procédé indirect, mais que d'autres éléments, au contraire, se concentrent dans la fonte et sont donc également concentrés dans les inclusions piégées dans le métal de la filière indirecte lors de l'affinage. Il est alors possible, en utilisant cette méthode statistique, d'émettre une probabilité 
d'appartenance à l'une ou l'autre des filières en comparant au référentiel la composition des inclusions mesurée sur un objet donné. Au-dessus d'une certaine probabilité $^{56}$, on peut retenir la filière candidate. Si cette probabilité est trop faible, l'attribution demeure incertaine.

\section{Étudier les procédés à travers leur production : les fers des cathédrales}

En utilisant l'approche qui vient d'être décrite, le procédé indirect a été pisté au sein des renforts métalliques employés pour la construction des grands monuments gothiques, dont l'érection s'est échelonnée du $\mathrm{XIII}^{\mathrm{e}}$ siècle au début de l'époque moderne, période qui croise celle de l'apparition et de la diffusion du nouveau procédé en Europe. Les recherches récentes ont en effet montré que d'importantes quantités de métal ont été utilisées dans les monuments gothiques médiévaux lors de leur construction $^{57}$. Ces fers ont pu être datés par analyse archéologique du bâti et plus récemment par la mise en place de la méthode radiocarbone suite à l'extraction des carbures de l'acier ${ }^{58}$.

Pour chacun des monuments étudiés et pour différentes périodes, la part des objets issus de chacune des filières a pu être déterminée par l'analyse des inclusions de scories piégées dans le métal des renforts métalliques. Ces analyses concernent plus de 250 armatures de fer ${ }^{59}$. La répartition des procédés est présentée (ill. 2-3-4 du cahier couleur) sous la forme de diagrammes circulaires (filière directe : bleu; filière indirecte : rouge). On observe qu'au XIII ${ }^{\mathrm{e}}$ siècle, la totalité des renforts, quelle que soit la localisation des monuments étudiés, a été réalisée avec du fer issu de la filière directe, ce qui est en bon accord avec les données textuelles et archéologiques pour les sites de production. Pour la première moitié du xIv siècle, le procédé direct reste majoritaire sur tous les monuments. Ainsi, il est le seul employé à Rouen, Troyes et Auxerre. En revanche, environ un tiers des fers analysés dans les églises de Liège est issu du procédé indirect. Cette occurrence devance légèrement les premières mentions textuelles pour le procédé dans la région, qui ne sont pas antérieures au milieu du XIV siècle. Pour la fin du XIV siècle, on note la présence des premiers fers indirects à la cathédrale d'Auxerre; ils constituent un quart des éléments analysés sur ce monument pour cette période. Cette fois, les sources écrites identifient l'arrivée du procédé indirect autour de cette région de manière concomitante. En revanche, les fers analysés à Liège pour le début $d u x^{e}$ siècle ne présentent plus trace du nouveau procédé : tous proviennent de la réduction directe en bas fourneaux. Il semble donc que, malgré l'arrivée précoce de la filière indirecte en Wallonie indiquée par les sources écrites et confirmée par l'archéométrie, l'emploi du fer direct perdure dans cette zone, même cent ans plus tard ${ }^{60}$. Le pro- 
cédé direct reste également le seul identifié à cette période sur les églises de la ville de Rouen. Pour cette dernière zone, les informations archéométriques sont cohérentes avec celles des sources écrites puisque la filière indirecte n'est pointée en Normandie qu'à la fin du $x^{e}$ siècle. Cinquante ans plus tard (période 14501500), le paysage sidérurgique est radicalement différent puisqu'on observe la présence de fers indirects sur l'ensemble des bâtiments étudiés, que ce soit à Metz, à Amiens et à Rouen, régions où les premières évidences de l'indirect datent $d u$ milieu $d u x^{e}$ siècle, mais aussi à Troyes où les plus anciennes mentions en Pays d'Othe sont cette fois plus précoces (fin du XIV siècle). Cette concordance presque parfaite des données de l'archéométrie avec celles issues des autres sources ne doit pas masquer, perduration du procédé direct dans les zones mêmes où la filière indirecte s'est diffusée. De même, sur l'ensemble du territoire étudié, dans la seconde partie du $\mathrm{Xv}^{\mathrm{e}}$ siècle, une part non négligeable des éléments métalliques identifiés sur les cathédrales reste issue de l'ancienne filière. Cette concomitance des deux filières sidérurgiques a pu être observée par l'archéologie en Lorraine et ce, jusqu'au $\mathrm{XvII}^{\mathrm{e}}$ siècle ${ }^{61}$, mais les résultats archéométriques permettent de généraliser cette observation à tout le nord du royaume de France et ce, au moins jusqu'au $\mathrm{XvI}^{\mathrm{e}}$ siècle puisque des fers directs sont encore identifiés à cette période (mais de manière cette fois très minoritaire) dans les églises troyennes ${ }^{62}$. Aux $\mathrm{XVII}^{\mathrm{e}}$ et $\mathrm{XVIII}{ }^{\mathrm{e}}$ siècle, le procédé indirect semble, cette fois, hégémonique sur l'ensemble des édifices étudiés pour cette période.

\section{Nouvelles considérations sur la diffusion de la filière indirecte}

La répartition des procédés observée sur les renforts des cathédrales n'est pas aussi tranchée en faveur du procédé indirect que ne le suggérait la floraison, contemporaine, de mentions écrites associées au nouveau procédé. On observe une concomitance des deux filières, et ce jusqu'à deux cents ans après les premières mentions écrites du procédé indirect. Précisons tout d'abord que les sources archéométriques étudiées ici concernent l'un des marchés du fer pour les époques considérées, celui des chantiers des grands monuments gothiques, qui ne représente qu'une part $\mathrm{du}$ fer produit et consommé. Il présente également plusieurs spécificités : les éléments de fer mis en œuvre sont des pièces le plus souvent assez peu travaillées, morphologiquement proches de la barre de fer issue de l'atelier de production, certains éléments peuvent néanmoins être issus du recyclage ou du remploi ${ }^{63}$, $d^{\prime}$ autres peuvent provenir, sans que ce soit une règle systématique, de sources assez distantes du chantier, comme cela a été démontré pour le Palais des Papes d'Avignon ${ }^{64}$ ou la cathédrale de 
Bourges $^{65}$. En ce qui concerne le recyclage, il est possible de le détecter dans le matériau analysé quand il résulte de la soudure de plusieurs éléments d'origines distinctes. Le remploi, consistant en une réutilisation sans modification de forme (ou avec des adaptations très mineures), est quant à lui détecté par la confrontation entre l'analyse du bâti et la datation radiocarbone des éléments ferreux. Cette récupération peut parfois être mise en évidence par les sources écrites comme, en 1432-1433, à la cathédrale de Troyes, où le serrurier forge des barreaux puis des croches de fer à partir de chaînes de fer récupérées par l'œuvre ${ }^{66}$ ou, en 1548-1549, à l'église Saint-Jean de Troyes, où le forgeron reçoit 226 livres de « viel fer » pour forger des agrafes ${ }^{67}$. Ainsi, s'il ne peut être exclu totalement pour les fers analysés dans les études auxquelles nous faisons référence dans cet article, le remploi ou le recyclage représente de manière certaine une part très mineure, soit parce que les fers étudiés ont été datés par radiocarbone ${ }^{68}$, soit parce qu'ils ne présentaient aucune trace de recyclage par assemblage d'éléments d'origines différentes. La question du lieu de production dont l'origine géographique peut être différente de celle de l'espace du marché doit également être considérée. Les études archéométriques récentes ont en effet pu montrer sur quelques monuments qu'une part des fournitures du chantier pouvait provenir d'espaces de production situés parfois à plus d'une centaine de kilomètres ${ }^{69}$. Ceci n'est cependant pas systématique et ne concerne jamais la majorité des fers ${ }^{70}$. En gardant en tête ces aspects des sources archéométriques, il est possible de les considérer pour reprendre le dos- sier de l'innovation technique au Moyen Âge, qui a fait et fait toujours l'objet de discussions importantes en histoire des techniques médiévales ${ }^{71}$.

Comme l'exprime Paul Benoit, « faire l'histoire de l'innovation, c'est d'abord établir une chronologie ${ }^{72} »$. À ce titre, l'archéométrie permet de pointer, avant toute reconnaissance écrite, les premières occurrences de l'innovation technique que représente le procédé indirect dans les églises de Liège par la présence de ses produits. Cependant, si, à Liège, $40 \%$ des objets analysés pour la période 13001350 sont issus de la nouvelle filière, les occurrences du nouveau procédé disparaissent pour les analyses de la période postérieure (1400-145073). Ceci ne suggère pas un processus qui bouleverse brutalement et intensément le marché ${ }^{74}$. En tout état de cause, l'impact du nouveau procédé semble, au moins pour ses débuts, moins important qu'attendu dans cette région pourtant pionnière dans son introduction. Par ailleurs, nous pouvons également pointer dans ces territoires la lenteur de la diffusion de l'innovation technique que constitue le procédé indirect $^{75}$, et surtout celle de l'utilisation des matériaux issus de ces nouvelles techniques. À la grande diversité régionale de la diffusion des nouvelles techniques, déjà mentionnée par les historiens, semble s'ajouter une certaine résistance au sein même des territoires où elle essaime $^{76}$. Cette résistance se retrouve encore quand le nouveau procédé indirect a diffusé vers le sud et notamment vers la Bourgogne : dans les cathédrales de Troyes et d'Auxerre, une bonne partie des éléments de renforts sont encore issus de la filière directe, alors même que la filière fonte est installée, comme en 
témoignent à la fois les sources écrites et les données archéométriques.

Le phénomène étudié par les sources archéométriques résulte de la convolution d'une réalité technique de production (procédés) et du marché qui écoule les produits, incluant facteurs techniques (qualité des matériaux en relation avec la nature de la demande), facteurs économiques, voire influence de réseaux politiques ou financiers ${ }^{77}$. La perduration de fers issus de la filière directe résulte-t-elle d'un choix conscient, par les hommes de l'art, d'un matériau présentant une qualité particulière? Il n'a pas été possible à l'heure actuelle de mettre en évidence une influence directe du procédé de réduction employé sur la qualité finale du matériau ${ }^{78}$. Au contraire, ce sont plutôt les étapes postérieures de la chaîne opératoire telles que le compactage de la masse brute sortie des bas fourneaux ou des foyers d'affinerie et le temps passé à la mise en forme de l'objet qui vont conditionner cette qualité. Celle-ci, au demeurant, peut être très variable en fonction des objets étudiés et, pour les fers de construction, dans la plupart de cas, aucune qualité spécifique ne semble requise par les constructeurs ${ }^{79}$. Considérons plus avant le cas particulier de la cathédrale d'Amiens, où le châ̂nage placé dans le triforium, en 1498, pour lequel les experts avaient préconisé l'usage de fer d'Espagne, est donc en toute probabilité issu du procédé direct. La majeure partie des éléments, massifs et standardisés, est issue du procédé indirect bien que, très spécifiquement, les fers utilisés pour chacune des extrémités - non standardisées - soient, quant à eux, exclusivement issus du procédé direct ${ }^{80}$. On pencherait, ici encore, non pas pour l'influence d'un choix technique, mais pour une signature de l'organisation et des vicissitudes du chantier. La majorité des fers du chaînage provient d'une grosse forge indirecte, probablement locale, vraisemblablement $\mathrm{du}$ fait $\mathrm{du}$ peu de disponibilité du fer d'Espagne. L'usage systématique de fer direct pour les extrémités reste à comprendre : changement d'approvisionnent à une phase spécifique du chantier correspondant à une commande particulière de la fabrique pour l'arrimage du chaînage au bâtiment, ou fournitures spécifiques aux artisans chargés de cet arrimage, arrivant sur le chantier avec leur propre fer destiné uniquement à cette opération? La question reste ouverte.

$\mathrm{Si}$, comme la conjonction des sources archéométriques et historiques tend à le prouver, on considère qu'au moins une partie du fer direct trouvé sur les monuments est bien d'origine locale, en l'état actuel de la recherche, l'hypothèse la plus probable pour la perduration de la filière directe pour un temps dans le nord de l'Europe ${ }^{81}$ serait alors liée à des raisons économiques, le coût d'installation de l'équipement lié au procédé indirect étant loin d'être négligeable, notamment en ces temps de crises qui marquent la fin du Moyen Âge ${ }^{82}$. L'exemple de Saint-Liébaut, première affinerie identifiée sur le sol du royaume de France, est à ce titre très évocateur : d'après les archives, l'affinerie ne semble pas survivre à la mort de son fondateur, Nicolas de Fontenay, en $1396^{83}$. Ce n'est que dans la seconde moitié $\mathrm{du} \mathrm{xv}^{\mathrm{e}}$ siècle que d'autres exemples manifestes d'usines à fer fonctionnant selon la filière indirecte sont à nouveau attestés avec certitude dans la région : «fer fondu » de 
l'abbaye du Reclus en 1451-1452 $2^{84}$, « fondoire » de la forge de Dilo en $1457^{85} \ldots$ Elles s'installent cette fois durablement sur le territoire et alimentent, entre autres, le marché des grands chantiers de construction. Les résultats de la présente étude, conjonction de l'analyse des textes et de la matière permettent d'étendre ces conclusions à une plus grande partie du nord-ouest de l'Europe, y compris dans certains foyers de l'innovation, pour proposer une évaluation quantitative de la diffusion du procédé indirect sur le temps long par l'étude d'un de ses marchés. D'après la chronologie suggérée par les sources écrites et archéométriques, il semble en effet que même si des facteurs politiques ou des périodes de relative embellie, notamment à la fin du XIv ${ }^{e}$ siècle, ont été propices à une introduction précoce du procédé dans certaines régions (Wallonie, Champagne, Nivernais...), celui-ci ne s'impose durablement qu'à la sortie de la guerre de Cent Ans, lors de la période de reprise économique marquant l'extrême fin du Moyen Âge et le début de la période moderne ${ }^{86}$.

Ces considérations permettent d'ancrer cette chronologie revisitée de la diffusion du procédé indirect à une échelle plus vaste de développement d'autres techniques à la fin du Moyen Âge. Tout comme la poudre, connue en Europe occidentale dès le $\mathrm{xIII}^{\mathrm{e}}$ siècle, mais dont les usages ne se répandent véritablement qu'au $\mathrm{Xv}^{\mathrm{e}}$ siècle avec le développement des armes à feu, ou le papier dont la fabrication est introduite en Occident dès la fin du XIII ${ }^{\mathrm{e}}$ siècle, mais qui n'offre une concurrence au parchemin qu'à partir du milieu $\mathrm{du} \mathrm{xv}^{\mathrm{e}}$ siècle en lien avec l'invention de l'imprimerie ${ }^{87}$, le procédé indirect ne semble connaître un véritable essor dans une partie de l'Europe du nordouest qu'à partir du milieu du $\mathrm{xv}^{\mathrm{e}}$ siècle, indépendamment de sa date de première introduction. Le marché étudié avec les fers de construction ne semblant pas ici faire appel à une qualité spécifique des produits recherchés, ce sont bien les conditions liées à l'introduction de l'innovation qui sont questionnées ici. Si $\mathrm{du}$ fer de réduction indirect vient désormais remplir les mêmes usages que le fer direct sur ces chantiers, c'est assurément par sa plus grande disponibilité sur le marché considéré. Certainement liée au développement des usages de la fonte - et par là même des hauts fourneaux -, cette disponibilité doit aussi être reliée aux facteurs économiques qui ont pu freiner ou favoriser le développement de ces usines à fer. $\mathrm{Ce}$ " poids de la conjoncture ", pointé par P. Benoit ${ }^{88}$, est certainement fondamental dans l'attribution de cette chronologie.

On peut ainsi remettre en question le réel impact du procédé indirect sur ce marché de la barre de fer avant la fin de la guerre de Cent Ans dans les territoires étudiés. Au sortir de la guerre, il est en revanche manifeste. L'étude des prix du fer acheté par les chantiers de construction troyens et rouennais montre qu'une lente chute des prix de la livre de fer s'opère à cette période : entre 1435 et 1480 à Troyes (de 12 à $6 \mathrm{~d}$.t. la livre environ), et à partir de 1455 jusqu'au début du $\mathrm{xvI}^{\mathrm{e}}$ siècle à Rouen (de 13 à 8 d.t. la livre) $)^{89}$. Ces fortes variations doivent certainement être reliées à l'installation définitive du nouveau procédé dont les usines permettent désormais la production de produits (fonte et fer) plus abondants et surtout à moindre coût. 
Cette brève présentation a montré comment les données physico-chimiques collectées sur les objets eux-mêmes, peuvent constituer une source à part entière, à même de dialoguer avec les textes ou les fouilles archéologiques. Dans le cas présent, les sources archéométriques témoignent d'un marché spécifique, celui du fer dans les grands chantiers gothiques. La perception de l'arrivée d'une innovation sur ce type de marché est cependant extrêmement complémentaire de celle qu'offrent les sources écrites qui révèlent la " première perception de l'innovation ${ }^{90} » \mathrm{du}$ point de vue, cette fois, de la production du métal, et de celle de l'archéologie qui, quant à elle, permet de saisir les procédés à travers l'étude des sites de production.

Les prochaines années devraient voir en France la poursuite et l'intensification de ces recherches interdisciplinaires autour de l'impact de l'apparition du procédé indirect sur les marchés du fer. Ceci passera par l'étude d'autres contextes d'utilisation du fer tels que ceux de l'armement ou de l'outillage agricole. Par ailleurs, comme nous l'avons montré, cette thématique de recherche sur les métaux ferreux est indissociable d'autres champs d'études tel que celui de la circulation des produits et de leur utilisation en fonction de différents facteurs (qualité, disponibilité, etc.).

Nous espérons enfin avoir rappelé comment ces approches interdisciplinaires, fondées sur une collaboration croisée et exigeante de chercheurs issus de disciplines complémentaires qui prend ses racines dans les recherches menées depuis les années 1960 autour de la compréhension des chaînes opératoires métallurgiques, sont arrivées à un degré d'intégration extrêmement élevé qui permet aujourd'hui d'éclairer avec une finesse inégalée des questions importantes de l'histoire des techniques et de l'économie.

\section{Notes}

1. Catherine Verna, « Réduction du fer et innovation : à propos de quelques débats en histoire sociale des techniques ", Médiévales, vol. Techniques : les paris de l'innovation, 2000, p. 79-95.

2. On pourra en trouver l'exposé détaillé dans plusieurs publications récentes : Philippe Dillmann, Stéphanie Leroy, Alexandre Disser, Sylvain BauvaIs, Enrique Vega, Philippe Fluzin, «Dernières avancées autour des études sur la production, la circulation et la datation des métaux ferreux archéologiques ", Les nouvelles de l'archéologie, $\mathrm{n}^{\circ} 138,2015$, p. 28-34; Stéphanie Leroy, Philippe DillmanN, Alexandre Disser, Maxime L'Héritier, Sylvain Bauvais, Philippe Fluzin, "Provenance et circulation des alliages ferreux ", dans Philippe Dillmann, Ludovic Bellot-Gurlet (dir.), Circulation des matériaux et des objets dans les sociétés anciennes, Paris, Éditions des archives contemporaines, 2014, p. 79-108; Stéphanie LEROY, Maxime L'HÉritier, Emmanuelle Delqué-Kolic, Jean-Pascal Dumoulin, Christophe Moreau, Philippe DillmanN, "Consolidation or initial design? Radiocarbon dating of ancient iron alloys sheds light on the reinforcements of French Gothic Cathedrals ", Journal of archaeological science, vol. 53, 2015, p. 190-201.

3. Dénommée comme telle car elle fait référence à la réaction chimique du même nom qui permet de transformer les oxydes de fer en fer métallique.

4. Philippe Fluzin, « Apport de l'archéométrie à la restitution de la chaîne opératoire en sidérurgie, matériaux et procédés. Études métallographiques ", in $6^{e}$ cours d'archéologie d'Andorre : l'obtention du fer par le procédé direct du IV au XIX siècle, Andorra la Vella, Govern d'Andorra, Ministeri de Cultura, 2002, p. 388-396; Philippe FluzIN, " La chaîne opératoire en sidérurgie : matériaux archéologiques et procédés. Apports des études métallographiques ", dans Aux origines de la métallurgie du fer en Afrique, Éditions de 1'UNESCO-BPI, Mémoires des peuples, 2002, p. 58-92; Radomir PleINER, Iron in archaeology. The european bloomery smelters, Prague, Archeologicky Ustav av Cr, 2000.

5. Ces conditions concernent la température et la pression partielle en dioxyde et monoxyde de carbone. On peut considérer que, dans les conditions 
du bas fourneau, la température à atteindre est supérieure à $1200^{\circ} \mathrm{C}$ et en moyenne de $1300^{\circ}-1400^{\circ} \mathrm{C}$.

6. $1534^{\circ} \mathrm{C}$ pour le fer, pouvant baisser jusqu'à $1400^{\circ} \mathrm{C}$ pour les aciers les plus carburés.

7. Cette masse est parfois appelée "loupe » ou « masse brute de réduction ».

8. L'acier étant du fer contenant entre 0,1 et $2 \%$ massiques de carbone. Un acier prend la trempe pour des teneurs en carbone supérieures à $0,3 \%$ massique.

9. Pour une historiographie du moulin aux époques médiévales, on pourra consulter l'introduction de Paul Benoit, Karine Berthier, " L'innovation dans l'exploitation de l'énergie hydraulique d'après le cas des monastères cisterciens de Bourgogne, Champagne et FrancheComté ", dans Patrice BесK (dir.), L'innovation technique au Moyen Âge, Actes du VI Congrès international d'archéologie médiévale (1 ${ }^{\text {er-5 }} 5$ octobre 1996, DijonMont Beuvray-Chenôve-Le Creusot-Montbard), Paris, Errance, 1998, p. 58-66.

10. Mais également d'autres éléments comme le silicium ou le phosphore.

11. Ceci donne naissance notamment à la poterie en fonte, mais également au boulet de fonte et, plus tardivement, à l'artillerie en fonte de fer, Paul Benoit, Philippe Dillmann, Philippe Fluzin, "Iron, cast iron and bronze. New approaches of the artillery history ", dans Gert MAGNUSSON (dir.), The importance of ironmaking, technical innovation and social change (Actes du colloque de Norberg), CSAUISPP, Jernkontorets Bergshistoriska Utskot, 1995, p. 241-257.

12. En Angleterre, dans l'abbaye cistercienne de Bordesley, Grenville Astill, A medieval industrial complex and its landscape: the metalworking watermills and its workshops of Bordesley Abbey, York, CBA, 1993, et en 1135 à Clairvaux, Catherine Verna, Paul BENOIT, «La sidérurgie de Clairvaux au Moyen Âge ( $\mathrm{XII}^{\mathrm{e}}-\mathrm{XV}^{\mathrm{e}}$ siècles) », dans Histoire de Clairvaux, Barsur-Aube, Association Renaissance de l'abbaye de Clairvaux, 1991, p. 90-92.

13. Enzo BARALDI, « Ordigni e parole dei maestri da forno bresciani e bergamaschi : lessico della siderurgia indiretta in Italia fra XII e XVII secolo », dans Philippe BRAUSTEIN (dir.), La sidérurgie alpine en Italie (XII ${ }^{e}$-XVII siècle), Rome, CEFR, 2001, p. 163-213.

14. Mathieu Arnoux, "Innovation technique, intervention publique et organisation du marché : aux origines du district sidérurgique de la Valteline (XIII'-XIV ${ }^{\mathrm{e}}$ siècles) », ibid., p. 215-232.

15. Enzo Baraldi, Manlio Calegari, « Pratica e diffusione della sideruria "indiretta" in area italiana (secc. XIII-XIV) », ibid., p. 93-162.

16. M. Arnoux, "Innovation technique..." op. cit.

17. Les témoins archéologiques d'une production au moins ponctuelle de fonte sur certains sites de production métallurgique dans la région du Val Gabbia et de Bienno sont notamment présentés dans Ph. Braunstein (dir.), La sidérurgie alpine en Italie..., op. cit. ; Costanza Cucini-Tizzoni, Marco Tizzoni, « Alle origini dell'altoforno : i siti della Val Gabbia e della Val Grigna a Bienneo in Valcamonica ", dans Pier Paolo Poggio, Carlo Simoni (éd.), Musei del ferro in Europa e in Italia. La ricerca e le esperienze di conservazione e valorizzazione, Atti del Convegno, Brescia-Tavernole sul Mella, septembre 2004, Brescia, 2006, p. 21-42. Les datations de certains ateliers prêtent toutefois à caution.

18. On pourra se référer à Philippe DillmanN, Liliane Perez, Catherine Verna, « Les aciers avant Bessemer ", dans Philippe Dillmann, Liliane Hilaire-Perez, Catherine Verna (dir.), L'acier en Europe avant Bessemer, Paris, CNRS-Université de Toulouse-Le Mirail, 2011, p. 7-69.

19. Jean-François Belhoste, « Mutations techniques et filières marchandes dans la sidérurgie alpine entre le $\mathrm{XIII}^{\mathrm{e}}$ et le $\mathrm{XVI}^{\mathrm{e}}$ siècle ", dans Ph. Braunstein (dir.), La sidérurgie alpine en Italie..., op. cit., p. 515-624.

20. Cet « impact de la soufflerie sur la qualité des fers » a été signalé, Catherine Verna, "Innovations et métallurgies en Méditerranée occidentale (XIII ${ }^{\mathrm{e}}$ $\mathrm{xV}^{\mathrm{e}}$ siècles) ", Anuario de estudios medievales, vol. 41, $n^{\circ} 2,2011$, p. 623-644.

21. Catherine Verna, " "Moulin à fer" : l'héritage de Bertrand Gille ", dans Aline Durand (dir.), Jeux d'eau. Moulins, meuniers et machines hydrauliques, $\mathrm{XI}^{e}$-XXe siècle. Études offertes à Georges Comet, Aix-enProvence, Publications de l'université de Provence, 2008, p. 273-286.

22. Jean-François BeLHoste, «Martinetus et fusina dans la sidérurgie alpine aux $\mathrm{xIII}^{\mathrm{e}}$ et $\mathrm{xIV}^{\mathrm{e}}$ siècles, réflexions philologiques ", dans Costanza CUCINITizzoni, Marco Tizzoni (dir.), Il ferro nelle Alpi. Giacimiento, miniere e metallurgia dall' Antichita al XVI secolo, Atti del convegno, Bieno, Commune di Bieno, 2000, p. 146-151; C. Verna, « "Moulin à fer" : l'héritage de Bertrand Gille », op. cit.

23. Les quantités de fonte trouvées parmi les déchets de la mouline de Castel Minier (Ariège) semblent révélatrices de ces tâtonnements techniques; communication orale Florian Téreygeol, fouille en cours. Rappelons également que la mouline et la forge à la catalane se caractérisent par leurs foyers ouverts permettant, peut-être, d'éviter une montée en température trop importante.

24. Ce grand nombre des foyers d'affinage était certainement nécessaire pour traiter par petites quantités la production de fonte du haut fourneau. Nils BJÖRKENSTAM, Sven ForNANDER, "Metallurgy and technology at Lapphyttan ", dans N. BJÜRKENSTAM, S. FoRNANDER (dir.), Medieval Iron in Society, Jernkontoret and Riksantikvarieämbetet, 
1985, p. 184-225; Gert MAGNUSSON, « Lapphyttan. An example of medieval iron production ", ibid., p. 21-33.

25. Robert B. Gordon, Terry S. Reynolds, "Medieval iron in Society-Norberg, Sweden, May 6-10, 1985 », Technology $\mathcal{E}$ Culture, vol. 27, n 1, 1986, p. 110-117.

26. Ainsi, pour les mêmes hauts fourneaux de la région de Bamsberg en Suède et à partir des mêmes références, E.E. Hjärthner-Holdar et C. Risberg n'hésitent pas à affirmer que les hauts fourneaux prédominent depuis le $\mathrm{XII}^{\mathrm{e}}$ siècle. L. Stenvik, plus prudent, fait remonter l'innovation au $\mathrm{XIV}^{\mathrm{e}}$ siècle. Eva HJärthNer-Holdar, Christina Risberg, "Technology of iron : choices and innovation ", Materials and manufacturing processes, vol. 24, $\mathrm{n}^{\circ}$ 9, 2009, p. 981-986; Lars F. STENVIK, « Iron production in Scandinavian archaeology ", Norwegian archeological review, vol. 36, $\mathrm{n}^{\circ} 2,2003$, p. 119-134.

27. Erik Myrstener, William Lidberg, Ulf Segerström, Harald Biester, David Damell, Richard BINDLER, «Was Moshyttan the earliest iron blast furnace in Sweden? The sediment record as an archeological toolbox ", Journal of archaeological science : reports, vol. 5, 2016, p. 35-44.

28. Albrecht JocKENHÖvel, Mittelalterliche Eisengewinnung im Märkischen Sauerland : archäometallurgische Untersuchungen zu den Anfängen der Hochofentechnologie in Europa, Rahden, Verlag Marie Leidorf, 2013; Hans Ludwig KNAU, Dietrich Horstmann, Manfred SONNECKEN, « La production de fonte dans la haute vallée de la Volme : contribution à l'histoire de la sidérurgie en Westphalie occidentale », dans Patrice BECK (dir.), L'innovation technique au Moyen Âge, VI congrès international de la société d'archéologie médiévale, Paris, Errance, 1996, p. 152-159; Manfred SÖNNECKEN, « Eisendarstellung im Flosshofen », Der Märker, 1972, p. 4-5.

29. H. L. Knau, D. Horstmann, M. Sonnecken, « La production de fonte dans la haute vallée de la Volme : contribution à l'histoire de la sidérurgie en Westphalie occidentale », op. cit.

30. A. JOCKENHÖVEL, Mittelalterliche Eisengewinnung im Märkischen Sauerland..., op. cit., p. 120 et 132 .

31. Ibid., p. 389.

32. Danielle Arribet-Deroin, Fondre le fer en gueuses au XVI siècle. Le haut fourneau de Glinet en pays de Bray (Normandie), thèse de doctorat en art et archéologie, Paris I Sorbonne, 2001.

33. C. Verna, "Moulin à fer" : l'héritage de Bertrand Gille», op. cit.

34. Brian G. AwTY, "The development and dissemination of the walloon method of ironworking ", Technology \& Culture, vol. 48, n 4, 2007, p. 783-803.

35. Ibid. Cette « polyvalence des ateliers métallurgiques » a été soulignée par C. VERNA, «Innovations et métallurgies en Méditerranée occidentale (XIII ${ }^{\mathrm{e}}$ $\mathrm{XV}^{\mathrm{e}}$ siècles) ", op. cit.

36. B.G. AwTY, «The development and dissemination of the walloon method of ironworking ", op. cit.

37. H. Collin, « Aux origines du bassin sidérurgique de Briey : les forges de la région de Moyeuvre à la fin $\mathrm{du} \mathrm{XIII}^{\mathrm{e}}$ siècle et au début du $\mathrm{XIV}^{\mathrm{e}}$ ", Actes du $98^{e}$ Congrès national des sociétés savantes (Philologie et histoire jusqu'en 1610), Saint-Étienne, 1973, Paris, Bibliothèque nationale, 1975, p. 61-80; Alain Girardot, " À propos d'un bail de forge en 1391 : fonte et forges hydrauliques en Lorraine au xv ${ }^{\mathrm{e}}$ siècle ", Annales de l'Est, 4, 1976, p. 275-284, repris par Jean-Marie YANTE, Le Luxembourg mosellan. Production et échanges commerciaux 1200-1560, Bruxelles, Académie royale de Belgique, 1996.

38. Koichi Horikoshi, L'industrie du fer en Lorraine, XII $-\mathrm{XVII}{ }^{e}$ siècles, Langres, Dominique Guéniot, 2008; Marc Leroy, " Autour de la "minette" : la perduration de la production de fer en bas fourneau en Lorraine à la fin du Moyen Âge ", dans P. BECK (éd.), L'innovation technique au Moyen Âge, op. cit., p. 145-150.

39. Joséphine RouILlARD, L'homme et la rivière : histoire du bassin de la Vanne au Moyen Âge (XII ${ }^{e}$ $X V I^{e}$ siècle), thèse de doctorat $\mathrm{d}$ 'histoire de l'université Paris 1 Panthéon-Sorbonne, 2003, p. 401. Le haut fourneau associé se trouverait à Valcon-sur-l'Ancre, situé à quelques kilomètres.

40. Ibid., p. 402; Jean-François Belhoste, Patrick LÉON, " Naissance d'une sidérurgie moderne aux confins du Berry (fin du XIV $\mathrm{XIV}^{\mathrm{e}}$ siècles) », dans Mélanges Jean-Yves Ribault, Cahiers d'archéologie et d'histoire du Berry, 1996, p. 45-51.

41. B.G. AwTY, "The development and dissemination of the walloon method of ironworking ", op. cit.

42. Jean-François Belhoste, Christiane ClaerRRoussel, François Lassus et al., La métallurgie comtoise, $\mathrm{XV}^{e}$-XIXe siècles. Étude du Val de Saône, Besançon, ASPRODIC, 1994, p. 21.

43. Ibid., p. 28.

44. K. Horikoschi, L'industrie du fer en Lorraine, XII ${ }^{e}$-XVII ${ }^{e}$ siècles, op. cit., p. 406.

45. Catherine Verna, Les mines et les forges des Cisterciens en Champagne méridionale et en Bourgogne du Nord, XII ${ }^{e}-X V^{e}$ siècles, Paris, AEDEH-Vulcain, 1995, p. 66-67.

46. Jean-François Belhoste, Yannick Lecherbonnier, Mathieu Arnoux (dir.), La métallurgie normande XII ${ }^{e}-\mathrm{XVII}$ siècles. La révolution du haut fourneau, Caen, L'Inventaire, 1991, p. 41-42.

47. Henry Cleere, David Crossley, The iron industry of the Weald, Chesterfield, Merton Priory Press, 1995. 
48. J.-F. Belhoste, « Mutations techniques...», op. cit., p. 515-624.

49. Jean-François BeLhoste, "L'implantation d'une sidérurgie bergamasque en Dauphiné au début du $\mathrm{xvII}^{\mathrm{e}}$ siècle ", dans Ninina Cuомo Di Caprio, Carlo Simoni (dir.), Dal basso fuoco all'altoforno, Atti del $1^{\circ}$ simposio Valle Camonica, Brescia, Grafo edizioni, 1991.

50. Danielle Arribet-Deroin, « Rythmes et pratiques de la métallurgie du fer aux $\mathrm{xIV}^{\mathrm{e}}$ et $\mathrm{XV}^{\mathrm{e}}$ siècles : les "bloomieres" de Tudeley (Kent) et de Byrkeknott (comté de Durham) ", Archéologie médiévale, vol. 40, 2010, p. 147-168; Catherine Verna, Le temps des moulines. Fer, technique et société dans les Pyrénées centrales (XIII - XVI siècles), Paris, Publications de la Sorbonne, 2001, p. 85. Il n'est toutefois pas certain que les premières moulines ariégeoises comme celle d'Albiès aient disposé d'une soufflerie hydraulique; elles sont en revanche bien équipées d'un gros marteau.

51. C. Verna, "Réduction du fer... ", op. cit., p. 79-95; Philippe Braunstein, Travail et entreprise au Moyen Âge, Bruxelles, De Boeck et Larcier, 2003.

52. Philippe Dillmann, Maxime L'Héritier, "Slag inclusion analyses for studying ferrous alloys employed in French medieval buildings : supply of materials and diffusion of smelting processes ", Journal of archaeological science, vol. 34, $\mathrm{n}^{\circ}$ 11, 2007, p. 1810-1823; Alexandre Disser, Philippe Dillmann, Catherine Bourgain, Maxime L'Héritier, Enrique Vega, Sylvain Bauvais, Marc LEROY, « Iron reinforcements in Beauvais and Metz Cathedrals : from bloomery or finery? The use of logistic regression for differentiating smelting processes ", Journal of archaeological science, vol. 42, $\mathrm{n}^{\circ} 1$, 2014, p. 315-333.

53. La réalisation de ces analyses se fait par spectrométrie EDS (Energy Dispersive Spectrometry) couplée au microscope électronique à balayage (MEB). Elle nécessite de réaliser un prélèvement sur l'objet; plus la taille de ce prélèvement est importante, plus les mesures seront représentatives, l'idéal étant de réaliser une coupe transversale complète de l'objet.

54. La méthode statistique utilisée est la régression logistique. Elle a été réalisée sur la composition des inclusions normalisées par rapports de logarithmes, voir A. Disser, Ph. Dillmann, C. Bourgain, M. L'Héritier, E. Vega, S. Bauvais, M. LEROY, "Iron reinforcements in Beauvais and Metz Cathedrals... », op. cit.

55. Scorie produite par le haut fourneau.

56. 0,9 dans A. Disser, Ph. Dillmann, C. Bourgain, M. L'Héritier, E. Vega, S. Bauvais, M. LEROY, "Iron reinforcements in Beauvais and Metz Cathedrals... », op. cit.

57. Pour de plus amples informations à ce sujet, on pourra se référer aux travaux spécifiques sur les différents monuments : Palais des Papes d'Avignon, Philippe Bernard, Philippe Dillmann, "Métal et architecture médiévale : l'exemple du Palais des Papes d'Avignon ", dans Nicolas Cucuzza, Maura Medri (dir.), Archeologie. Studi in onore di Tiziano Mannoni, Bari, 2003, p. 279282; Philippe Bernardi, Philippe Dillmann, «Stone skeleton or iron skeleton: The provision and use of metal in the construction of the Papal Palace at Avignon in the $14^{\text {th }}$ century ", dans Robert BoRK (dir.), De re metallica. The uses of metal in the Middle Ages, Aldershot, Asghate, 2005, p. 297-315; Philippe Bernardi, Philippe Dillmann, " L'impiego del metallo nel cantiere trecentesco del Palazzo dei Papi di Avignone ", dans Vittorio Franchetti Pardo (dir.), Arnolfo di Cambio e la sua epoca, Roma, Viella, 2007, p. 239-348; Philippe Dillmann, Philippe BERNARDI, " Premiers résultats métallographiques sur les tirants de fer du Palais des Papes d'Avignon. Éléments de réflexion sur la qualité et la provenance des matériaux ferreux utilisés dans la construction monumentale au Moyen Âge ", dans Jean-Pierre Sosson, Isabelle Paquay (dir.), Au-delà de l'écrit. Les hommes et leurs vécus matériels au Moyen Âge à la lumière des sciences et des techniques. Nouvelles perspectives, Louvain-la-Neuve, Brepol, Typologie des sources du Moyen Âge occidental Hors Série, 2003, p. 241-279), cathédrales et églises de Rouen et de Troyes (Maxime L'HÉRItIER, « L'utilisation du fer à la cathédrale de Rouen à l'époque médiévale ", dans Jean-Pierre WATte (dir.), Haute Normandie archéologique, CRAHN, 2004, p. 69-78; Maxime L'Héritier, Philippe Dillmann, Paul Benoit, « Premiers résultats métallographiques sur les fers de construction de la cathédrale Notre-Dame de Rouen ", dans Jean-Paul Hervieu, Gilles Desire-Dit-Gosset, Éric BARRE (éd.), Les arts du feu en Normandie, Actes du $39^{\circ}$ congrès organisé par la Fédération des sociétés historiques et archéologiques de Normandie, Caen, Annales de Normandie, 2005, p. 287-314; Maxime L'Héritier, "L'utilisation du fer dans l'architecture gothique. Approche méthodologique à travers les villes de Rouen et Troyes ", dans Arnaud Timbert (dir.), L'homme et la matière. L'emploi du fer et du plomb dans l'architecture gothique, Paris, Picard, 2009, p. 61-74; Maxime L'Héritier, Philippe Dillmann, Paul Benoit, «Iron in the building of gothic churches : its role, origins and production using evidence from Rouen and Troyes », Historical metallurgy, vol. 44, $\mathrm{n}^{\circ}$ 1, 2010, p. 21-35; Maxime L'Héritier, Philippe Dillmann, "Fer ou acier? Caractérisation des alliages ferreux utilisés dans la construction des églises gothiques au Moyen Âge et à la période moderne. L'exemple de Troyes et de Rouen ", dans Ph. Dillmann, L. HilairePerez, C. Verna (dir.), L'acier en Europe avant 
Bessemer, op. cit., p. 263-284), cathédrales d'Amiens, de Soissons et de Beauvais (Philippe Dillmann, «De Soissons à Beauvais : le fer des cathédrales de Picardie, une approche archéométrique », dans A. Timbert (dir.), L'homme et la matière. L'emploi du fer et du plomb dans l'architecture gothique, op. cit., p. 93-112), d'Auxerre (Sylvain Aumard, Philippe Dillmann, Maxime L'HérITIER, « Le métal selon l'archéologue et l'archéomètre ", dans Sylvain AumARd (dir.), Saint-Étienne d'Auxerre. La seconde vie d'une cathédrale, Auxerre, CEM-Picard, 2011), de Coutances (Maxime L'HÉRITIER, « Réflexion sur les usages du fer à la cathédrale de Coutances et dans l'architecture gothique normande ", dans Pierre Bouet, Gilles DésIRÉ Dit Gosset, Françoise LATY (dir.), La cathédrale de Coutances. Artet histoire, Bayeux, Orep éditions, 2012, p. 39-56), de Metz (Alexandre Disser, Production et circulation du fer en Lorraine ( $V I^{e}$ s. av. J.-C.-XVe s. ap. J.-C.), université de Technologie de Belfort-Montbéliard, 2014), de Chartres (Maxime L'Héritier, Émeline Lefebvre, Adrien Arles, Philippe DillmanN, Bernard Gratuze, « Oculi des baies hautes du chœur. Étude archéologique et archéométrique des éléments métalliques », dans Arnaud Timbert (dir.), Chartres. Construire et restaurer la cathédrale (XIII'-XXI siècle), Villeneuve d'Asq, Presses universitaires du Septentrion, 2014, p. 307320; Émeline Lefebvre, Maxime L'Héritier, «De l'emploi du fer dans la structure de la cathédrale de Chartres. Approche qualitative et quantitative ", ibid., p. 287-306), basilique de Saint-Denis (Maxime L'HÉritier, "Fer et plomb sur le chantier », dans Pascal Delannoy (dir.), Saint-Denis. Dans l'éternité des rois et des reines de France, Strasbourg, La nuée bleue, 2015), donjon du château de Vincennes (Jean Снapelot, " Le donjon et son enceinte. Une résidence royale ", Les dossiers d'archéologie - Vincennes, vol. 289, 2003, p. 60-73), églises liégeoises (Christophe Maggi, Gaspard Pages, Anne Mertens, Patrick HoFfSUMMER, « Utilisation et technique de production $\mathrm{du}$ fer et $\mathrm{du}$ bois dans les charpentes de comble mosanes : premiers jalons d'une évolution du $\mathrm{XII}^{\mathrm{e}}$ au XVIII ${ }^{\mathrm{e}}$ siècle », Archéosciences, revue d'archéométrie, vol. 36, 2012, p. 95-115).

58. Le carbone donnant naissance à ces carbures provient du charbon de bois utilisé pour la réduction du minerai. Il est possible d'extraire une partie de ce carbone et de doser la quantité de ${ }^{14} \mathrm{C}$ qu'il contient, ce qui permet de le dater.

59. S. Aumard, Ph. Dillmann, M. L'Héritier, " Le métal selon l'archéologue et l'archéomètre », op. cit.; Ph. Dillmann, M. L'Héritier, « Slag inclusion analyses... », op. cit.; Ph. DillmanN, «De Soissons à Beauvais... ", op. cit. ; C. MAgGI, G. PAgES, A. Mertens, P. Hoffsummer, «Utilisation et technique de production du fer et du bois dans les char- pentes de comble mosanes : premiers jalons d'une évolution du XII ${ }^{\mathrm{e}}$ au XVIII ${ }^{\mathrm{e}}$ siècle », op . cit.

60. Des analyses en cours dans notre laboratoire révèlent $q u^{\prime} \mathrm{au} \mathrm{xv}^{\mathrm{e}}$ siècle, une part importante des fers de renfort du beffroi de l'hôtel de ville de Bruxelles est également constituée de fers de réduction directe.

61. M. Leroy, "Autour de la "minette" », op. cit., p. $145-150$.

62. Une part de recyclage ou de remploi est aussi probablement à prendre en compte. Cette question est discutée plus bas; Maxime L'HérITIER, Alexandre Disser, Stéphanie Leroy, Philippe Dillmann, "Récupérer et recycler les matériaux ferreux au Moyen Âge : des textes à la matière ", dans Yves Henigfeld, Philippe Husi, Fabienne RAvoire (dir.), L'objet au Moyen Âge et à l'époque moderne : fabriquer, échanger, consommer et recycler. Actes du XI congrès de la Société d'archéologie médiévale, moderne et contemporaine, Caen, Presses universitaires de Caen, sous presse.

63. Philippe Dillmann, Maxime L'Héritier, «Récupération et remploi du fer pour la construction des monuments de la période gothique ", dans Jean-François BERnARd, Philippe BERnARd, Daniela Esposito, Philippe Dillmann, Laura Foulquier, Rossana MANCINI (dir.), Il reimpiego in architettura. Recupero, trasformazione, uso, Rome, École française de Rome, 2009, p. 157-176.

64. Stéphanie Leroy, Circulation au Moyen Âge des matériaux ferreux issus des Pyrénées ariégeoises et de la Lombardie. Apport du couplage des analyses en éléments traces et multivariées, université de technologie de Belfort-Montbéliard, 2010.

65. Maxime L'Héritier, Stéphanie Leroy, Philippe DillmanN, Bernard Gratuze, "Characterisation of slag inclusion in iron objects ", dans Laure Dussubieux, Mark GolitKo, Bernard Gratuze (dir.), Recent advances in laser ablation ICP-MS for archaeology, Springer, 2017, p. 213-228.

66. Archives départementales [désormais AD] Aube, G 1562, fol. $137 \mathrm{r}^{\circ}$.

67. AD Aube, 15 G 57, fol. $79 \mathrm{r}^{\circ}$.

68. C'est le cas à Bourges, Metz, Beauvais.

69. Philippe Dillmann, Maxime L'Héritier, Alexandre Disser, Stéphanie Leroy, Enrique VEgA, "Produire, échanger, utiliser les métaux ferreux au Moyen Âge : nouveaux éclairages interdisciplinaires ", dans Y. HeNigfeld et al. (dir.), L'objet au Moyen Âge et à l'époque moderne, op. cit.

70. À la cathédrale de Bourges par exemple, seuls $30 \%$ des fers sont avec certitude issus de productions lointaines. Il s'agit là d'un maximum sur l'ensemble des chantiers étudiés. Les mentions de provenance dans les sources écrites, quand elles sont disponibles, vont dans le même sens. Pour le chantier de la cathédrale de Troyes, à l'exception 
de trois mentions de "fer d'Espagne ", l'ensemble des provenances font référence à des fers de production locale ou régionale, Maxime L'Héritier, Philippe Dillmann, Paul Benoit, «Iron in the building of gothic churches : its role, origins and production using evidence from Rouen and Troyes ", Historical metallurgy, 44, 1, 2010, p. 21-35. Dans le cas du Châtillonais, O. Chapelot estime à environ $75 \%$ la part des fers de production locale sur les chantiers, Odette CHAPELOT, « Les ouvriers du métal en Bourgogne à la fin du Moyen Âge : l'exemple du Châtillonais ", dans Odette CHAPELOT, Paul BenoIT (éd.), Pierre et métal dans le bâtiment au Moyen Âge, Paris, EHESS, 1985, p. 305-308.

71. Paul Benoit, Philippe Lardin, "Les paris de l'innovation ", Médiévales, n 39, 2000, p. 5-13; Philippe Braunstein, «L'innovation dans les mines et la métallurgie européenne ( $\mathrm{XIV}^{\mathrm{e}}-\mathrm{XVI}^{\mathrm{e}}$ siècles) ", Bulletin de l'Association française des historiens économistes, vol. 15, 1982, p. 1-17; Jean-Marie Pesez, "Introduction : le Moyen Âge est-il un temps d'innovation technique? ", dans P. Bеск (éd.), L'innovation technique au Moyen Âge, op. cit., p. 11-14; C. Verna, «Réduction du fer... », op. cit.

72. P. Benoit, Ph. LaRdin, « Les paris de l'innovation $»$, op. cit.

73. Malheureusement, à Liège, il n'a pas été possible d'analyser des objets postérieurs à 1450 .

74. Si l'on considère la théorie de Schumpeter, explicitée par P. Benoit et Ph. Lardin : " Pour qu'il $\mathrm{y}$ ait innovation il faut que le changement soit d'importance, qu'il modifie les conditions de la production et du marché. » Ceci ne semble pas être le cas pour le marché spécifique des fers des grands monuments gothiques de la région de Liège.

75. Cette lenteur de la diffusion des nouvelles techniques est également relevée par P. BENOIT, Ph. LaRdin, op. cit. Parfois, cependant, le temps de l'adoption d'une nouveauté peut être assez rapide, par exemple, 10 ans pour l'installation d'une horloge mécanique dans toutes les villes d'Europe entre 1370 et 1380, Ph. BraunsteIn, Travail et entreprise au Moyen Âge, op. cit., 2003, p. 31.

76. Cette résistance est différente de celle révélée par d'autres auteurs où le procédé direct n'a pas cédé le pas à la filière fonte jusqu'au $\mathrm{XIX}^{\mathrm{e}}$ siècle. Il s'agit notamment du sud de la France, de l'aire pyrénéenne et de la Catalogne, C. Verna, Le temps des moulines..., op. cit.

77. C. VERNA, «Innovations et métallurgies... », op. cit.

78. M. L’Héritier, Ph. Dillmann, "Fer ou acier?...», op. cit.

79. Ibid. Maxime L'Héritier, Philippe Dillmann, Ivan Guillot, Philippe Bernard, " Première approche du comportement mécanique des fers de construction anciens ", dans Françoise FleurY, Laurent BARIDOn, Antonella Mastrorilli, Rémy
Mouterde, Nicolas Reveyron (dir.), Les temps de la construction. Processus, acteurs, matériaux, Actes du deuxième congrès francophone d'histoire de la construction, Lyon 29-31 janvier 2014, Paris, Picard, 2016, p. 555-567. On citera comme rare contre-exemple mis en évidence à ce jour celui du Palais des Papes d'Avignon où la mise en œuvre de barres d'acier a été révélée par l'archéométrie, Ph. Dillmann, Ph. Bernardi, "Premiers résultats métallographiques... ", op. cit.; Ph. Bernardi, Ph. Dillmann, "Stone skeleton... », op. cit., et les sources écrites et celui de la cathédrale d'Amiens, détaillé ci-dessous.

80. Ph. Dillmann, «De Soissons à Beauvais... », op. cit.; Émeline LeFEBVRE, « Les tirants de fer de la cathédrale Notre-Dame d'Amiens ", dans Arnaud Timbert (dir.), L'emploi du fer et du plomb dans l'architecture gothique, Paris, Picard, 2009, p. 141-47.

81. La situation dans le sud de l'Europe et en particulier dans la péninsule ibérique et le sud de la France est différente. Ici le procédé indirect perdure et l'innovation passe par l'emploi de la force hydraulique et son amélioration pour la ventilation sans atteindre la fusion du métal. Jean CANTELAUBE, La forge à la catalane dans les Pyrénées ariégeoises, une industrie à la montagne (XVII - XIXe siècle), Toulouse, CNRS/université de Toulouse-Le Mirail, 2005;

C. Verna, Le temps des moulines..., op. cit.

82. Pour des raisons chronologiques, elle ne peut strictement être liée à la crise du bois que connaît $l^{\prime}$ Europe occidentale de la fin du $\mathrm{XII}^{\mathrm{e}}$ siècle au milieu $\mathrm{du}$ XIII ${ }^{\mathrm{e}}$ siècle en raison de la réduction des espaces boisés.

83. J. RouilLard, L'homme et la rivière..., op. cit., p. 406.

84. M. L'Héritier, Ph. Dillmann, P. Benoit, "Iron in the building of gothic churches », op. cit.

85. J. Rouillard, L'homme et la rivière..., op. cit., p. $408-409$.

86. P. Benoit, Ph. Lardin, « Les paris de l'innovation », op. cit.

87. Paul Benort, «Au four et au moulin : innovation et conjoncture ", dans P. Beck (dir.), L'innovation technique..., op. cit., p. 293-301.

88. Ibid.

89. Maxime L'HéRITIER, L'utilisation du fer dans l'architecture gothique : les cas de Troyes et Rouen, thèse de doctorat d'archéologie, université Paris 1, 2007, p. 764-766; Philippe LARDIN, Les chantiers du bâtiment en Normandie orientale (XIV ${ }^{e}-\mathrm{XVI}^{e} \mathrm{~s}$.), les matériaux et les hommes, Villeneuve d'Ascq, Presses universitaires du Septentrion, 2001, p. 270-272. Cette période de baisse des prix est suivie d'une hausse importante au $\mathrm{XVI}^{\mathrm{e}}$ siècle, qui a pu être reliée à la hausse des prix $\mathrm{du}$ bois dont l'industrie du fer fait une très importante consommation; D. Arribet-Deroin, Fondre le fer en gueuses, op. cit., p. 169.

90. C. Verna, Le temps des moulines..., op. cit., p. 150. 